\title{
FALSE LEMMAS IN HERBRAND
}

\section{BY BURTON DREBEN, ${ }^{1}$ PETER ANDREWS AND STÅL AANDERAA}

Communicated by A. M. Gleason, May 2, 1963

In his thesis of 1929 (see [1]) Herbrand showed how to generate effectively from an arbitrary quantificational schema $S$ a sequence of quantifier-free expressions $R_{1}(S), R_{2}(S), \cdots$, called reductions (réduites) of $S$, and said that $S$ has property $C$ of order $p$ if $R_{p}(S)$, the $p$ th reduction of $S$, is truth-functionally valid. (Throughout this paper "effective" will mean "primitive recursive." When reductions are defined below, the reader should observe that a schema has property $C$ of order $p$ only if it has property $C$ for all orders $>p$.) Herbrand also gave a set of rules of inference, called rules of passage, the main point of which is to permit an arbitrary schema to be transformed into an equivalent prenex one, and then argued:

(I) If, for any given $p=1,2,3, \cdots$, a schema $S$ has property $C$ of order $p$, then any schema $T$ obtained from $S$ solely by the rules of passage also has property $C$ of the same order $p$.

(II) If $S$ and $W$ are any schemata such that $S$ has property $C$ of order $p$ and the schema $7 S \vee W$ has property $C$ of order $q$, then the schema $W$ has property $C$ of order $\max (p, q)$.

(Assertion (I) is Lemma 3 in $\S 3$ of Chapter 5 of [1], and assertion (II) is Lemma 3 in $\$ 5$ of the same chapter.)

On the basis of (I) and (II) Herbrand readily established what he called his Fundamental Theorem, varying versions of which are now known as Herbrand's Theorem. More specifically, for a particular axiomatic system tC of quantification theory which we shall soon describe, Herbrand constructed on the basis of (I) and (II) two effectively computable functions $\phi$ and $\psi$ such that

(1) if $D(S)$ is any derivation in $\mathfrak{H C}$ of a quantificational schema $S$, then $\phi(D(S))$ is a truth-functionally valid reduction of $S$, and

(2) if $R_{p}(S)$ is any truth-functionally valid reduction of $S$, then $\psi\left(R_{p}(S)\right)$ is a derivation of $S$ in FC in which no use is made of modus ponens.

But (I) and (II) are false. Indeed, after the necessary background material has been sketched, we shall show that for each $p \geqq 3$

(a) there are schemata $S$ and $T$ such that $T$ comes from $S$ by just one application of one rule of passage and $S$ has property $C$ of order 3 but $T$ has property $C$ of no order $<p$, and

1 With partial support from NSF Grant GP-228. 
(b) there are schemata $S$ and $W$ such that the schemata $S$ and $T S \vee W$ each have property $C$ of order 3 but $W$ has property $C$ of no order $<p$.

The system FC takes negation, alternation, and both universal and existential quantification as primitive, and has as axioms all quantifier-free tautologies. Rules of inference are modus ponens, generalized simplification (i.e. replace an occurrence of a well-formed part $P \vee P$ in a derivable schema by an occurrence of $P$ ), universal generalization, existential generalization, and the following twelve rules of passage:

Rules of passage. Let $W$ and $Z$ be any schemata and let $v$ be any variable not occurring in $Z$. For $h=1, \cdots, 6$, let $J_{h} ; K_{h}$ be the $h$ th of the following six ordered pairs of schemata:

1. $\urcorner \forall v W ; \exists v\urcorner W$,

2. $7 \exists v W ; \forall v\urcorner W$,

3. $\forall v W \bigvee Z ; \forall v(W \bigvee Z)$,

4. $Z \vee \forall v W ; \forall v(Z \bigvee W)$,

5. $\exists v W \bigvee Z ; \exists v(W \vee Z)$,

6. $Z \bigvee \exists v W ; \exists v(Z \bigvee W)$.

Also let $J_{6+h} ; K_{6+h}$ be $K_{h} ; J_{h}$, the converse of $J_{h} ; K_{h}$. Then a schema $T$ will be said to come from a schema $S$ by one application of the ith rule of passage $(i=1, \cdots, 12)$ if $T$ is the result of replacing one occurrence of $J_{i}$ in $S$ by one occurrence of $K_{i}$. The occurrence of $J_{i}$ in question will be called the $n u b$ of the application.

The system $\mathfrak{H C}$ just described differs from the main system of Herbrand's thesis (see pp. 31-32 of [1] and corrections noted on pp. 22-23 of [2]) in having the rule of generalized simplification in place of the rule of simplification, that is, in having the rule of generalized simplification in place of the rule that if $S \vee S$ is derivable then $S$ is derivable. (Modus ponens is not eliminable from the main system. See p. 117 of [1].) Also, implicit in Herbrand's formulation of $x^{2}$ is the rule of alphabetic change of bound variables.

To define the reductions of a schema we first note that any quantificational schema can be transformed effectively into an equivalent rectified schema, that is, into a schema which contains no variable occurring both free and bound, and which contains no two occurrences of quantifiers with the same variable. Now let $S$ be any such rectified schema. Call a bound variable $v$ and its quantifier $Q v$ in $S$ restricted (restreinte) either if $Q v$ is existential and lies within the scopes of an even number of negation signs (zero counts as even) or if $Q v$ is universal and lies within the scopes of an odd number of negation signs. Call all other variables and their quantifiers (if any) 
in $S$ general (gếnérale); and call any general variable in $S$ simple if it is either a free variable or its quantifier lies within the scope of no restricted quantifier. Let $y_{1}, \cdots, y_{t}$ be all the distinct general variables in $S$; for each $i=1, \cdots, t$, let $x_{i, 1}, \cdots, x_{i, m_{i}}$ be, in some fixed order, the variables of the $m_{i}\left(m_{i} \geqq 0\right)$ distinct restricted quantifiers within whose scopes lies the quantifier $Q y_{i}$; call $y_{i}$ the functional term of $y_{i}$ if $y_{i}$ is simple; call $y_{i}\left[x_{i, 1}, \cdots, x_{i, m_{i}}\right]$ the functional term of $y_{i}$ if $y_{i}$ is not simple; call $x_{i, 1}, \cdots, x_{i, m_{i}}$ the argument terms of $y_{i}\left[x_{i, 1}, \cdots, x_{i, m_{i}}\right]$; and let the functional form $F(S)$ of $S$ be the expression obtained from $S$ by deleting all general quantifiers in $S$ and replacing each general variable by its functional term at each of its remaining occurrences.

For each rectified schema $S$ we define the finite sets $D_{1}^{S}, D_{2}^{S}, D_{3}^{S}, \ldots$ inductively thus:

(1) The set $D_{1}^{S}$ contains just the numeral " 1 ".

(2) The set $D_{m+1}^{S}$ contains all members of $D_{m}^{S}$, and in addition all those expressions which are obtainable from some functional term in $F(S)$ by replacing each argument term (if any) of the functional term by some member of $D_{m}^{S}$.

Note that all simple variables in $S$ belong to the set $D_{2}^{S}$; that if $S$ contains no general variable then $D_{m}^{S}$ is $D_{1}^{S}$ for all $m$; that if $S$ contains no nonsimple general variable then $D_{m}^{S}$ is $D_{2}^{S}$ for all $m \geqq 2$; and that if $S$ contains at least one nonsimple general variable then for each $m$ the set $D_{m+1}^{S}-D_{m}^{S}$ is not empty. (Herbrand called the set $D_{m+1}^{S}-D_{m}^{S}$ the $(m+1)$ st field $($ champ $)$.)

Now define the reduction $R_{p}\left(F(S)\right.$ ) of $F(S)$ over $D_{p}^{S}$ inductively by associating with each well-formed part $W$ of $F(S)$ a reduction $R_{p}(W)$ over $D_{p}^{S}$ as follows:

(1) If $W$ contains neither quantifiers nor truth-functional connectives then $R_{p}(W)$ is $W$.

(2) If $W$ is the negation of a well-formed part $A$ of $F(S)$ then $R_{p}(W)$ is the negation of $R_{p}(A)$.

(3) If $W$ is the alternation of well-formed parts $A$ and $B$ of $F(S)$ then $R_{p}(W)$ is the alternation of $R_{p}(A)$ and $R_{p}(B)$.

(4) If $W$ is a restricted quantifier $Q x$ followed by its scope $Z$ and $R_{p}(Z)$ is the reduction of $Z$ over $D_{p}^{S}$, then let $\sum_{x} R_{p}(Z)$ be the alternation (taken in some fixed order) of all those distinct expressions obtainable by replacing all occurrences of $x$ in $R_{p}(Z)$ (including its occurrences as an argument term) by occurrences of a member of $D_{p}^{s}$. Construct $\prod_{x} R_{p}(Z)$ similarly using conjunction instead of alternation. Then $R_{p}(W)$ is $\sum_{x} R_{p}(Z)$ if $Q x$ is an existential quantifier and is $\prod_{x} R_{p}(Z)$ if $Q x$ is a universal quantifier. 
Finally, if $S$ is an arbitrary schema, let $S^{\prime}$ be a rectified schema equivalent to $S$ (and effectively obtained from $S$ ), and define $R_{p}(S)$, the reduction of $S$ over $D_{v}^{S}$, or briefly the pth reduction of $S$, to be $R_{p}\left(F\left(S^{\prime}\right)\right)$.

For an instructive example of the above notions, let $S$ be

$$
\urcorner[\forall x G x \vee \top \forall y N y] \vee \forall y_{1} G y_{1} \vee\right\urcorner \forall x_{1} N x_{1}
$$

and let $T$ be

$$
\urcorner \forall x[G x \vee \top \forall y N y] \vee \forall y_{1} G y_{1} \vee \top \forall x_{1} N x_{1}
$$

Then $S$ has property $C$ of order 2, but $T$ has property $C$ of no order earlier than 3. For $D_{2}^{S}$ is the set $\left\{1, y, y_{1}\right\}$ and $R_{2}(S)$ is the truthfunctionally valid expression

$$
\left.7\left[\left(G 1 \& G y \& G y_{1}\right) \vee\right\urcorner N y\right] \vee G y_{1} \vee \top\left(N 1 \& N y \& N y_{1}\right) \text {. }
$$

However, since $D_{2}^{T}$ is the set $\left\{1, y[1], y_{1}\right\}, R_{2}(T)$ is the truth-functionally nonvalid expression

$$
\begin{array}{r}
7\left[(G 1 \vee \top N y[1]) \&(G y[1] \vee \top N y[y[1]]) \&\left(G y_{1} \vee \top N y\left[y_{1}\right]\right)\right] \\
\left.\vee G y_{1} \vee\right\urcorner\left(N 1 \& N y[1] \& N y_{1}\right) .
\end{array}
$$

$B$ ut then the schemata $S$ and $T$ refute (I). For $T$ comes from $S$ by one application of the third rule of passage.

Now say that an effectively computable function $\chi$ is a strong analyzing function for a given rule of passage if, for any $p=1,2,3, \cdots$, whenever an arbitrary schema $S$ has property $C$ of order $p$ then any schema $T$ coming from $S$ by one application of the rule of passage has property $C$ of order $\chi(p)$. Herbrand's assertion (I) is that the identity function is a strong analyzing function for each rule of passage. But we have just seen that this is false. Indeed, as the following complex example shows, there is no strong analyzing function for either the third or fourth rule of passage.

For each $p=1,2, \cdots$, let $A_{p}$ be the schema

$\forall y_{2} \forall y_{3} \cdots \forall y_{p+1}\left[T G y_{2} y_{3} \vee\right\urcorner G y_{3} y_{4} \vee \cdots \vee \top G y_{p-1} y_{p}$

let $S_{p}$ be the schema

$$
\left.\vee T M y_{2} y_{p+1} \vee \exists x_{8} M y_{p} x_{8}\right] \text {; }
$$

$\left.\left.\left.A_{p} \vee \exists x_{1} \exists x_{2}\right\urcorner[\urcorner G x_{1} x_{2} \vee \forall z\right\urcorner M x_{1} x \vee \exists y_{1} M x_{2} y_{1}\right] ;$

and let $T_{p}$ be the schema

$$
A_{p} \vee \exists x_{1} \exists x_{2} \top\left[\top G x_{1} x_{2} \vee \forall x\left(\top M x_{1} x \vee \exists y_{1} M x_{2} y_{1}\right)\right] .
$$

Then the schema $S_{p}$ has property $C$ of order 3, and the schema $T_{p}$ has property $C$ of order $p$ but of no earlier order. 
Undoubtedly, the reader will have noticed that the present counterexample to (I) results, as does the earlier one, from a single application of the third rule of passage with a negatively occurring nub (i.e., with a nub lying within the scope of an odd number of negation signs). Now we shall say that an application of a rule of passage is crucial if it is an application either of the third or of the fourth rule of passage with a negatively occurring nub. We shall say this because only with respect to crucial applications does Herbrand err. In all other applications of the rules of passage (I) holds; that is, for all rules of passage except the third and the fourth and for these except when applied with negatively occurring nubs the identity function is a strong analyzing function. (The heart of Herbrand's error is on pp. 103-105 of [1]. Given schemata $S$ and $T$ which result from one another by rules of passage, Herbrand attempts to embed the sets $D_{p}^{S}$ into the sets $D_{\nu}^{T}$ in a special way. However, he fails to verify that his embedding is well defined and possesses the properties he requires; for crucial applications of rules of passage the verification is not possible.)

From the falsity of (I) with respect to crucial applications follows at once the falsity of (II). In the second counterexample to (I) we saw that for each $p=1,2,3, \cdots$, although $S_{p}$ has property $C$ of order $3, T_{p}$ has property $C$ of earliest order $p$. But now consider the schema $7 S_{p} \vee T_{p}$. For each $p$, it also has property $C$ of order 3 . So (II) is false. Note, however, that the schemata $S$ and $T$ occurring in the first counterexample to (I) do not give a counterexample to (II). Hence to obtain a counterexample relatively easy to check, let $S$ and $T$ be respectively the schemata

\section{$\left.\forall y_{1} M y_{1} \bigvee \exists x_{1}\right\rceil G x_{1} \bigvee \top\left[\forall x_{2} M x_{2} \bigvee \exists y_{2} N y_{2}\right] \bigvee \top\left[\forall x_{3} \top N x_{8} \bigvee \top \forall y_{3} G y_{3}\right]$}

and

$\forall y_{1} M y_{1} \bigvee \exists x_{1} \top G x_{1} \bigvee \top \forall x_{2}\left[M x_{2} \bigvee \exists y_{2} N y_{2}\right] \bigvee \top \forall x_{3}\left[\top N x_{3} \bigvee \top \forall y_{3} G y_{3}\right]$

Here $T$ comes from $S$ by two crucial applications of the rules of passage, and has property $C$ of earliest order 4 . But the schemata $S$ and $\urcorner S \vee T$ have property $C$ of orders 2 and 3 respectively.

For tracing the deeper implications of the falsity of (I) it proves convenient to extend the notion of a strong analyzing function. So let $g$ be any $n$-premised quantificational rule of inference $(n=1, \cdots)$. A strong analyzing function for $\mathfrak{g}$ is any $n$-placed effectively computable function $\chi$ such that, for any given $n$-tuple $p_{1} ; p_{2} ; \cdots ; p_{n}$ of numbers and any given $n$-tuple $S_{1} ; S_{2} ; \ldots ; S_{n}$ of arbitrary schemata, if for each $i=1, \cdots, n$ the schema $S_{i}$ has property $C$ of order 
$p_{i}$, then the number $\chi\left(p_{1}, \cdots, p_{n}\right)$ is the order to which any schema $T$ will have property $C$ if $T$ comes from $S_{1} ; S_{2} ; \cdots ; S_{n}$ by a single (appropriate) application of $\mathscr{g}$.

Thus (II) asserts that for the 2-premised rule of modus ponens there is a strong analyzing function, namely, the function $\max (p, q)$ where the first premise has property $C$ of order $p$ and the second premise has property $C$ of order $q$. But the second counterexample to (II) shows that this function cannot be a strong analyzing function for modus ponens, and the first counterexample to (II) shows that no function can be.

When we look at Herbrand's argument for (II) we see that it fails at only one point: in assuming, by relying on (I), that a schema has property $C$ of order $p$ only if a prenex form obtainable from the schema by the rules of passage has property $C$ of order $p$ (p. 115 of [1]). This false assumption, which we shall label A(I), is made by Herbrand at each key point in Chapter 5 of his thesis. From it he quickly proves (p. 114 of [1]) that the successor function is a strong analyzing function for the rule of existential generalization and that the identity function is a strong analyzing function for both the rules of universal generalization and generalized simplification. (Herbrand considers only simplification, but his discussion extends at once to generalized simplification.) But then Herbrand has completed his argument for the first and more difficult part of his theorem. Indeed, since each quantifier-free tautology has property $C$ of order 1 , Herbrand has shown on the basis of (I) and $\mathrm{A}(\mathrm{I})$ that if $D(S)$ is any derivation in $\mathfrak{H C}$ of an arbitrary schema $S$ and if existential generalization has been applied $k$ times in $D(S)$, then the $(k+1)$ st reduction of $S$ is truth-functionally valid; i.e., $\phi(D(S))=R_{k+1}(S)$. Moreover, the only flaw in Herbrand's argument (p. 113 of [1]) for the second part of his theorem is that it also begins with the same false assumption $\mathrm{A}(\mathrm{I})$.

Hence Herbrand's argument can be repaired and his Fundamental Theorem established if we can find a weak analysing function for crucial applications of rules of passage; that is, if we can find an effectively computable 2-placed function $\zeta$ such that for any given schema $S$ and any given number $p=1, \cdots$, if $S$ has property $C$ of order $p$, then the number $\zeta(S, p)$ is the property $C$ order of any schema $T$ coming from $S$ by one application of the third or fourth rule of passage with a negatively occurring nub. For given such a function $\zeta$ we can construct an effectively computable 3-placed function $\theta$ such that if $S$ is any schema having property $C$ of order $p$ and 
$T$ is any prenex schema obtained from $S$ solely by the rules of passage, then $T$ has property $C$ of order $\theta(S, p, T)$. And then, using the information supplied by $\theta$ wherever Herbrand uses $A(I)$, we get a correct proof. In particular, we see that the rules of universal and existential generalization as well as the rule of generalized simplification have indeed the strong analyzing functions Herbrand claimed for them. But of course the rule of modus ponens does not. Rather, for it we get what we may again call a weak analyzing function, that is, an effectively computable 4-placed function $\gamma$ such that for any given schemata $S$ and $T S \vee T$ and any given numbers $p$ and $q$ if $S$ has property $C$ of order $p$ and $7 S \vee T$ has property $C$ of order $q$, then the schema $T$ has property $C$ of order $\gamma(S, T, p, q)$. However, and this is what Herbrand really wanted to achieve, we can still construct an effectively computable function $\psi$ such that for any $S$ and any $p$ if $R_{p}(S)$ is truth-functionally valid, then $\psi\left(R_{p}(S)\right)$ is a normal form derivation of $S$ in $\mathcal{H}$, that is, a derivation in which no applications of either the third or fourth rules of passage or modus ponens ever occur. So a Herbrand-type proof of Herbrand's Theorem turns squarely upon finding a weak analyzing function for crucial applications of rules of passage. And such a function follows at once from (III) below.

(III) Let the schema $S$ have property $C$ of order $p$; let $T$ be any schema coming from $S$ by one application of the third or the fourth rule of passage with a negatively occurring nub; and let there be $r(\geqq 0)$ restricted quantifiers within whose scopes the nub of the application lies. Then

(a) the schema $T$ has property $C$ of order $2 p-1$ when $r=0$,

(b) the schema $T$ has property $C$ of order

$$
1+\sum_{k=1}^{k=p-1} \prod_{i=1}^{i=k}\left[1+(n(i))^{r}-(n(i-1))^{r}\right] \text { when } \quad r>0,
$$

where $n(i)$ is the cardinality of $D_{i}^{S}$, and where $\sum$ and $\Pi$ are ardinary arithmetic summation and multiplication. (The proof of (III), by Dreben, appears in his introduction to [3].)

Note that in the first counterexample to (I) the number $r$ is 0 and in the second it is 2 . Note further that only when $r>0$ do crucial applications lack strong analyzing functions.

We are grateful to Alonzo Church, W. V. Quine, Hao Wang, John Denton, Robert Fenichel, and David Ragozin for helpful comments. 


\section{REFERENCES}

1. Jacques Herbrand, Recherches sur la theorie de la demonstration, Travaux de la Société des Sciences et des Lettres de Varsovie, Classe III sciences mathématiques et physiques, no. 33 (1930).

2. - Sur le probleme fondamental de la logique mathematique, Comptes Rendus des séances de la Société des Sciences et des Lettres de Varsovie, Classe III sciences mathématiques et physiques, no. 24 (1931).

3. John van Heijenoort, Editor, Jacques Herbrand, Ecrits Logiques, Presses Universitaires, Paris (to appear)

HARVARD UNIVERSITY AND

PrINCETON UNIVERSITY

\section{SIMPLY INVARIANT SUBSPACES 1}

BY T. P. SRINIVASAN

Communicated by Edwin Hewitt, March 18, 1963

Let $L^{1}, L^{2}$ denote respectively the spaces of summable and square summable functions on the circle group and $H^{1}, H^{2}$ their subspaces consisting of those functions whose Fourier coefficients vanish for negative indices. A closed subspace $M$ of $L^{1}$ or $L^{2}$ is "invariant" if

$$
x M \subset M
$$

and "simply invariant" if the above inclusion is strict, where $\chi$ is the character

$$
\chi(x)=e^{i x} .
$$

The structure of simply invariant subspaces is known, namely, they are precisely the subspaces of the form $q H^{1}$ or $q H^{2}$ (respectively) where $q$ is a measurable function of modulus 1 a.e. Beurling [1] first proved this for subspaces $M \subset H^{2}$; for $M \subset H^{1}$, this is due to de LeeuwRudin [5]; for $M \subset L^{2}$, due to Helson-Lowdenslager [3] and for $M \subset L^{1}$, due to Forelli [2]. In [3] Helson-Lowdenslager also gave a simple proof of the $H^{2}$ case, free of function theoretic considerations. Using their arguments Hoffman [4] extended this result to simply invariant subspaces of $\boldsymbol{H}^{2}(\mathrm{dm})$ defined over logmodular algebras. In this paper we prove this result for simply invariant subspaces of $L^{2}(d m)$ and $L^{1}(d m)$ over logmodular algebras; the results of the previous authors follow as a corollary. The proofs of the previous authors

\footnotetext{
1 This work was done while I held a visiting appointment at the University of California, Berkeley.

I thank Professors Helson and Ju-kwei Wang for the useful discussions I had with them.
} 